\title{
A Secure Control Protocol for USB Mass Storage Devices
}

\author{
Fuw-Yi Yang, Tzung-Da Wu, and Su-Hui Chiu
}

\begin{abstract}
The Universal Serial Bus (USB) has become the most popular interface standard for hardware connection, and there has been an explosion in the number of USB peripheral devices. External USB storage devices, in particular, are finding widespread application. Unfortunately, because USB affords high transmission speeds and is extremely convenient, many workplaces and businesses have prohibited the use of USB devices to prevent confidential data from being extracted from computer systems via USB ports. However, this compromises the convenience of the USB connection and demonstrates a lack of flexibility in management. Therefore, finding a way to take the considerations of both convenience and management into account has become a significant issue. This study proposes a secure control protocol which provides mutual authentication and key agreement between client and server to solve this problem $^{1}$.
\end{abstract}

Index Terms —Diffie-Hellman key exchange, digital signature, key agreement, mutual authentication, storage device, Universal Serial Bus (USB).

\section{INTRODUCTION}

The rapid development of computer and information industry in recent years has produced various new peripheral devices that can connect to a business computer terminal, especially via a USB port, which has become a standard component of current computer hardware due to its convenience and ease of connectivity. The main advantage of the USB port is that it offers a single interface connection between the computer and various devices, such as hard disks, flash drives, printers, keyboards, and PDA devices.

Unfortunately, because USB has high transmission speeds and is so convenient, using one is like passing data through an unprotected gate. For example, flash drives or other storage devices expose commercial intellectual property and confidential business data to the risk of theft. Therefore, effective control of these storage devices has become a significant issue for information security. If USB usage is comprehensively banned, then physical transmission between computers for various computer peripherals will be chaotic as

${ }^{1}$ Fuw-Yi Yang is with the Computer Science and Information Engineering Department, Chaoyang University of Technology, Taichung, Taiwan (e-mail: yangfy@cyut.edu.tw).

Tzung-Da Wu is with the Computer Science and Information Engineering Department, Chaoyang University of Technology, Taichung, Taiwan (e-mail: s9727621@cyut.edu.tw).

Su-Hui Chiu is with the Office of Accounting, Chaoyang University of Technology, Taichung, Taiwan (e-mail: suhui @cyut.edu.tw). before. If USB usage is not banned, then confidential data will probably be lost. Thus, balancing the convenience of the USB port with its lack of security is the most difficult USB management issue; confidential data must be secure, but access to USB devices must not be infringed upon. In this paper, we have proposed a control protocol which implements user authentication and key agreement to effectively govern file transmission via the USB port.

In this proposed protocol, a user needs a password to achieve mutual authentication with an authentication server. Without this legal authentication procedure, the system will not permit access to a USB port. After the authentication succeeds, the user and the server negotiate a key, which is used to encrypt/decrypt a file transmitted via the USB port. Thus the bits transmitted in the communication channels and the files stored in the USB devices are all protected by the negotiated key. For remote authentication, we adopt the digital signature scheme proposed by Schnorr in 1989 [1]. The most important feature of this scheme is that the parameters $e$ and $s$ are calculated and then saved, and when signifying, only one modular multiplication and one modular addition are required. This method can reduce costs, compared to other digital signature schemes. For the signature length, Schnorr's length is $|e|+|s|$; thus, if the order of the cyclic group is about $2^{160}$ and the output length of the hash function is 160 bits, then the signature length is 320 bits, i.e., 160 bits +160 bits $=320$ bits. In the RSA (Rivest, Shamir, and Adleman) cryptosystem [2], the signature length is $|n|=1024$ bits; in the ElGamal system [3], the signature length is $2 \times|p|=2048$ bits. Schnorr's digital signature scheme has a comparatively short length, and in practice is suitable for an environment requiring high efficiency and convenience.

For key agreement we used the key exchange agreement proposed by Whitfield Diffie and Martin Hellman in 1976 [4]. The objective of the Diffie-Hellman key exchange is for both sides to obtain the same session key by sharing some secret information via exponential and modular computations when the two sides try to communicate. Subsequently, the key can be used for encrypting a message for transmission. However, this protocol is subject to men-in-the-middle attacks. Suppose an attacker exists between the sending end and the receiving end. The attacker poses as the sending end to transmit a public key to the receiving end; without identity confirmation, the receiving end cannot ensure that this message is sent from the sending end. Many scholars have recently presented solutions to this problem. The most widely adopted of these is that of using a user password for identity confirmation on the two 
sides [5]-[7]. We developed a system based on password verification that combined Schnorr's digital signature scheme and the Diffie-Hellman key exchange agreement to realize system security and convenience.

The structure of this paper is as follows: Chapter 2 introduces our protocol and system environment; Chapter 3 focuses on a discussion and security analysis of our proposed protocol; and Chapter 4 presents our conclusion.

\section{Proposed Scheme}

To effectively prevent the theft of information via USB storage devices, we designed a control protocol to provide user authentication and key agreement. The system environment settings, mutual authentication and key agreement are described in detail below.

\section{A. System Environment Overview}

To manage a USB storage device effectively, any file transfer via a USB interface is restricted unless a user first passes a legal authentication procedure. A user wanting to transmit a file to a storage device via a USB interface must input a user name and password to verify legality. Then, the user is able to access the USB storage device.

In the user verification process, the user and the authentication server will generate a session key, which is used for encrypting all files transmitted to the storage device via the USB interface. Thus, all files stored on the USB storage device are encrypted. After the files are encrypted and transmitted, USB access will be restricted until the next successful verification. If users want to decrypt the file on the USB storage device later, they must pass the same verification procedure and obtain the same agreement key to acquire the original file. This agreement key is established for each filename and user identity; different users or files generate different keys. In addition, the system will delete the agreement key temporarily saved on the user end after the file is encrypted/decrypted, thus ensuring key independence and system security.

This proposed protocol has the following three characteristics:

1. Only users verified to be legal can access the USB storage device.

2. Even if a confidential file on a storage drive is stolen, the file cannot be decrypted without a key.

3. Even if a legal file owner wants to malevolently store confidential data on a storage device and distribute it to another person, the owner cannot obtain the corresponding agreement key for decryption as long as the authentication server suspends this user account. Consequently, the original file is secure. Figure 1 shows the flow chart of the entire control protocol.

Before introducing the control protocol, we define the parameters and symbols used in this paper.

- $p, q$ : Two large primes $p$ and $q$, where $q \mid p-1$

- $g, G: g$ is an element chosen from $Z_{p}^{*}$ and having an order of $q$; $G$ is the cyclic group generated by $g$

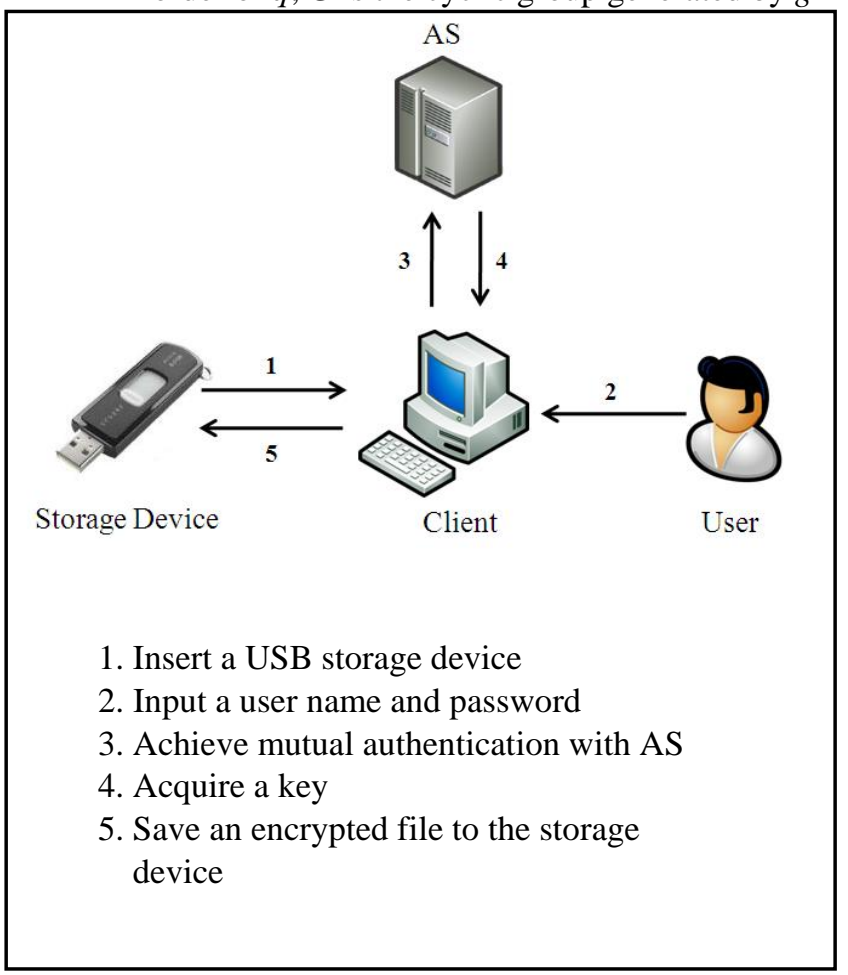

Fig. 1. Flow chart of control protocol

- id, $p w$ : User account (user name) and password

- $x, Y$ : Server's private key and public key; $Y=g^{x}$ mod $p$

- $h(\cdot), H(\cdot)$ : One way collision-resistant hash functions; $h(\cdot)$ maps arbitrarily long strings to strings of fixed length, and $H(\cdot)$ maps to elements of the cyclic group $G$

- \|: Concatenate operate

- $F_{n}$ : Filename for encryption

- File: File for encryption

- $E_{K}[\cdot]$ : Symmetric encryption function with respect to a key $K$

- $D_{K}[\cdot]$ : Symmetric decryption function with respect to a key $K$

\section{B. Registration Phase}

In our protocol, a user needs to pass through a registration phase before using the system. During the registration phase, the user first inserts a USB storage device and then chooses one set of $i d$ and $p w$. The $p w$ is substituted into the one way hash function to calculate $h p w=H(p w)$, and then id and $h p w$ are sent to the authentication server. When receiving this registration message, the server will choose a random number $k$ and generate $r=h p w^{k} \bmod p$ and $r_{1}=g^{k} \bmod p$. It then computes $e=h\left(i d\|r\| r_{1}\right)$, use its private key $x$ to calculate $s$ $=(k-e \cdot x) \bmod q$, and save $(e, r, s)$ to the user's storage device. After receiving the triplet $(e, r, s)$, the user checks whether $e$ is equal to $h$ (id $\|r\| g^{s} y^{e} \bmod p$ ). A valid check concludes the registration phase. Data transmission in this phase is done under a secure channel. In addition, in order to 
avoid password guessing attacks, the protocol will force the user to choose a password with sufficient complexity. Figure 2 shows the registration phase, where Client and AS denote user and authentication server respectively.

\begin{tabular}{|c|c|c|}
\hline Client & & AS \\
\hline \multicolumn{3}{|l|}{$h p w=H(p w)$} \\
\hline $\begin{array}{l}e^{?}=h\left(i d\|r\| g^{s} \cdot y^{e} \bmod p\right) \\
\text { save } e, r, s\end{array}$ & $e, r, s$ & $\begin{array}{l}\text { Random } k \\
r=h p w^{k} \bmod p \\
r_{1}=g^{k} \bmod p \\
e=h\left(i d\|r\| r_{1}\right) \\
s=k-e \cdot x \bmod q\end{array}$ \\
\hline
\end{tabular}

Fig. 2. Registration phase

\section{Verification and Data Encryption Phase}

After completing the registration phase, and when accessing the USB storage device, the user needs to achieve mutual authentication with the authentication server using the id and the $p w$, which generates an encryption key. The communication procedure is described in detail below. Figure 3 shows the full procedure of verification and data encryption phase.

\section{Step 1:}

The user attaches the USB storage device to a computer through a normal procedure and inputs the correct id and $p w$. At this moment, the user (client) will use $p w$ to calculate $h p w$ using the one way hash function $H(\cdot)$. Then the user chooses a random number $r_{C}$ and calculates $u=h p w \cdot y^{r} C \bmod p$ and $w=$ $g^{r} C \bmod p$. Finally, the user will send messages of $\left\{F_{n}, i d, e, s\right.$, $u, w\}$ to the authentication server.

\section{Step 2:}

After receiving $\left\{F_{n}, i d, e, s, u, w\right\}$, the authentication server will use its long term private key $x$ to calculate $h p w=u / w^{x}$ $\bmod p$ and $k=s+e \cdot x \bmod q$. The authentication server also utilizes its self-generated $h p w$ and $k$ to calculate $r=h p w^{k}$ mod $p$. Then, the authentication server will employ parameters it generated to verify whether $e^{?}=h\left(i d\|r\| g^{s} y^{e} \bmod p\right.$ ). If yes, then the user in this communication is legal. If not, the communication is terminated. Next, the authentication server generates random number $r_{S}$ and calculates $z=g^{r}$ mod $p$ and the session key $s k=w^{r_{S}}=g^{r_{C}} \cdot r_{S} \bmod p$.

Subsequently, the authentication server will use the received file name $F_{n}$ and the long-term private key to calculate $n=h\left(x \| F_{n}\right)$, and perform symmetric encryption on $n$, denoted as $E_{s k}[n]$, with respect to the session key sk. Finally, the authentication server calculates a verification message MAC $=h(h p w \|$ id $\|n\| s k \| w)$ and sends the generated message $\left\{E_{s k}[n], z, M A C\right\}$ to the user.

\section{Step 3:}

After receiving the message $\left\{E_{s k}[n], z, M A C\right\}$, the user uses the random number $r_{C}$ and the received $z$ to calculate a session key $s k$, and utilizes this session key sk to decrypt $n$, denoted as $D_{s k}\left[E_{s k}[n]\right]$. Next the user will verify whether $M A C \stackrel{?}{=} h(h p w \|$ id $\|n\| s k \| w)$. If yes, then mutual authentication is achieved between the user and the authentication server, and the user will calculate $a=h(i d \| n)$ and generate an encryption key $K$ using the equation $K=(Y)^{a}=g^{x \cdot a} \bmod p$.

\section{Step 4:}

After the user and the authentication server complete these steps, the encryption key $K$ can be calculated by $K=g^{x \cdot a}$ mod $p$. When a user wants to access the storage device via the USB interface, this encryption key, can be used to encrypt the File, i.e., as $E_{K}[$ File $]$, to protect the file and provide private and secure access to the USB device. For file decryption, the user needs to undergo the same verification steps and obtain the same key $K$ to decrypt the file $\left(D_{K}\left[E_{K}[\right.\right.$ File $\left.\left.]\right]\right)$ when accessing it on the USB device.

\section{SeCURITy ANALysis}

In this chapter, we present our control protocol for USB storage devices, which not only realizes mutual authentication, key agreement, and file protection but also resists some general attacks.

\section{A. Completeness}

We hope that the proposed system will effectively prevent any confidential file loss via USB removable storage devices. Thus, in our protocol design, file transfer via the USB interface is blocked unless the user first passes the remote server authentication procedure, and all files transmitted to the storage device are encrypted with the key $K$. Consequently, if users want to obtain the original file on another computer, they must pass the same verification procedure and acquire the same key $K$ for decrypting the file. The key $K$ is generated in accordance with the user id and the filename $F_{n}$, so different files or users produce different keys.

Therefore, when discovering that someone has saved a confidential file to a USB storage device, a security administrator only needs to suspend the user account, and the user will not be able to acquire the same key $K$ for decrypting the file and cannot access the original file.

\section{B. Off-Line Password Guessing Attack}

Let's assume an attacker tries to use an off-line password guessing attack to access a user password. First, the attacker might capture the ciphertext $(u, w)$ and verification message $M A C=h(h p w\|i d\| n\|s k\| w)$, where $u=h p w \cdot y^{r} C \bmod p$ and $w=g^{r} C \bmod p$. Without the long term private key $x$, using 
$(u, w)$ to calculate $h p w$, he will face the hard task of solving the 


\begin{tabular}{|c|c|c|}
\hline Client & & AS \\
\hline $\begin{array}{l}h p w=H(p w) \\
\text { Random } r_{\mathrm{C}} \\
u=h p w \cdot y^{r C} \bmod p \\
w=g^{r C} \bmod p \\
\\
\text { sk= } z^{r} C \bmod p \\
\text { decryption } D_{s k}\left[E_{s k}[n]\right] \\
M A C ? h(h p w \| \text { id }\|n\| s k \| w) \\
a=h(i d \| n) \\
K=Y^{a}=g^{x \cdot a} \bmod p \\
E_{K}[\text { File }]\end{array}$ & $\begin{array}{r}F_{n}, i d, e, s, u, w \\
\stackrel{E_{s k}[n], z, M A C}{\longleftarrow}\end{array}$ & $\begin{array}{l}h p w=u / w^{x} \bmod p \\
k=s+e \cdot x \bmod q \\
r=h p w^{k} \bmod p \\
e^{?}=h\left(i d\|r\| g^{s} y^{e} \bmod p\right) \\
\text { Random } r_{S} \\
z=g^{r} S \bmod p \\
s k=w^{r} S \bmod p \\
n=h\left(x \| F_{n}\right) \\
\text { encryption } E_{s k}[n] \\
M A C=h(h p w\|i d\| n\|s k\| w)\end{array}$ \\
\hline
\end{tabular}

Fig. 3. Verification and encryption phase

discrete logarithm problem. For the verification message $M A C$, the attacker would have difficulty guessing the password $p w$ (and consequently $h p w=H(p w)$ ) correctly because a random session key sk will be generated for each verification message in our protocol. The attacker cannot calculate $s k=g^{r} C \cdot r_{S}$ mod $p$ without knowing one of the random numbers $r_{C}$ and $r_{S}$. Thus, our protocol can resist offline password guessing attacks.

\section{Replay Attack}

In our protocol, the user and the authentication server choose random numbers, $r_{C}$ and $r_{S}$, and use these numbers to generate the session key $s k$. Therefore, when an attacker tries to use a captured wiretap login message $\left\{F_{n}, i d, e, s, u, w\right\}$ to execute a replay attack, the authentication server will return $\left\{E_{s k}[n], z, M A C\right\}$. At this moment, without $r_{C}$, the attacker cannot calculate the session key $s k=g^{r} C \cdot r_{S} \bmod p$ for decrypting $E_{s k}[n]$. Without obtaining $n$, the attacker cannot calculate the encryption key $K$ and read the original file. Thus, the replay attack is thwarted.

\section{Stolen-Verifier Attack}

When a USB storage device is lost or stolen, even if the attacker can acquire the verification factors $(e, r, s)$, the proposed protocol will not be compromised. In this system, where $e=h\left(i d\|r\| r_{1}\right), r=h p w^{k} \bmod p$, and $s=k-e \cdot x \bmod$ $q$, without the private server key $x$, the attacker cannot obtain any confidential data from $(e, r, s)$. Furthermore, even though the attacker tries to employ $(e, r, s)$ to pass the server verification, the user password $p w$ is still required; therefore, our protocol can resist the stolen-verifier attack.

\section{Conclusions}

In this paper, we have proposed a secure and efficient control protocol for USB ports. The protocol employs a remote authentication server to verify legal users and uses the Diffie-Hellmen key technique to protect the privacy of a file transmitted to a storage device. We have further proved that our protocol can resist some general attacks. In terms of protocol communication costs, realizing mutual authentication requires only two rounds of communication sessions. Therefore, the proposed protocol provides an effective control protocol for USB storage devices which is both secure and efficient.

\section{REFERENCES}

[1] C. P. Schnorr, "Efficient identification and signatures for smart cards," Journal of Cryptology, Vol. 4, pp. 161-174, 1991.

[2] R. Rivest, A. Shamir, and L. Adleman, "A method for obtaining digital signatures and public-key cryptosystems," Communications of the ACM, Vol. 21, No. 2, pp. 120-126, 1978. 
[3] T. A. ElGamal, "A public-key cryptosystem and a signature scheme based on discrete logarithms," IEEE Transactions on Information Theory, Vol. 31, No. 4, pp. 469-472, 1985.

[4] W. Diffie and M. Hellman, "New directions in cryptography," IEEE Transactions on Information Theory, Vol. 22, No. 6, pp. 644-654, 1976.

[5] Tzung-Her Chen, Wei-Bin Lee, "A new method for using hash functions to solve remote user authentication," Computers \& Electrical Engineering, Vol. 34, No. 1, pp. 53-62, 2008.

[6] Hyun Sook Rhee, Jeong Ok Kwon, and Dong Hoon Lee, "A remote user authentication scheme without using smart cards," Computer Standards \& Interfaces, Vol. 31, No. 1, pp. 6-13, 2009.

\section{BIOGRAPHIES}

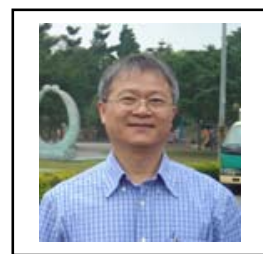

Fuw-Yi Yang received the BS and MS degree in the Department of Electronic Engineering from National Taiwan University of Science and Technology, Taipei, Taiwan, and the Ph.D. degree in the Department of Applied Mathematics, National Chung Hsing University, Taichung, Taiwan. He is currently an associate professor with the Department of Computer Science and Information Engineering, Chaoyang University of Technology, Taichung, Taiwan. He is a member of the Chinese Cryptology and Information Security Association (CCISA) and Taiwanese Association for Consumer Electronics (TACE). His research interests include computer cryptography, network security, and information security.

Tzung-Da Wu received the BS degree in the Department of Computer Science and Information Engineering, Chaoyang University of Technology. $\mathrm{He}$ is currently pursuing his MS degree in the Department of Computer Science and Information Engineering, Chaoyang University of Technology. His research interests include network security and information security.

Su-Hui Chiu received the BS degree in the Department of Accounting and Information, Overseas Chinese University, Taichung, Taiwan, and the MS degree in the Department of Industrial Engineering and Management, Chaoyang University of Technology. She is currently the director of the Accounting Office, Chaoyang University of Technology. Her research interests include financial management and information security. 